\title{
Causes, management and complications of severe adult dehydration in the emergency room
}

\author{
Abdulrahman Mohammad Ghafouri ${ }^{1 *}$, Shurouq Hamzah Arkoubi², \\ Talal Maqboul Al-Amri ${ }^{3}$, Afaf Matar Alshamari ${ }^{4}$, Mohammed Ali Alqahtani, $^{5}$ \\ Zainalaabdeen Sayyar Alyami ${ }^{6}$, Eiman Mohammad Alnemer ${ }^{7}$, Ahmed Musallam Almujayrishi ${ }^{8}$, \\ Najdi Ibrahem Najdi', Nawwaf Jamaan Althobaiti ${ }^{10}$, Hanan Yahia Lamfon ${ }^{11}$, \\ Rayyan Abdullah Alyahya ${ }^{12}$, Sally Ahmed Agbawi ${ }^{13}$
}

\author{
${ }^{1}$ Department of Emergency Medicine, King Faisal Hospital, Mecca, Saudi Arabia \\ ${ }^{2}$ Department of Internal Medicine, National Guard Health Affairs, Medina, Saudi Arabia \\ ${ }^{3}$ Department of Internal Medicine, King Fahad General Hospital, Medina, Saudi Arabia \\ ${ }^{4}$ Department of Internal Medicine, King Abdulaziz Specialist Hospital, Aljouf, Saudi Arabia \\ ${ }^{5}$ College of Medicine, King Khalid University, Abha, Saudi Arabia \\ ${ }^{6}$ Department of Emergency Medicine, King Khlaid Hospital, Najran, Saudi Arabia \\ ${ }^{7}$ Department of Emergency Medicine, King Fahad Hospital, Alahsa, Saudi Arabia \\ ${ }^{8}$ Department of Emergency Medicine, Heraa General Hospital, Mecca, Saudi Arabia \\ ${ }^{9}$ Department of Emergency Medicine, Ohud Hospital, Medina, Saudi Arabia \\ ${ }^{10}$ Department of Emergency Medicine, King Abdulaziz Specialist Hospital, Taif, Saudi Arabia \\ ${ }^{11}$ General Practiitoner, Ministry of Health, Jeddah, Saudi Arabia \\ ${ }^{12}$ College of Medicine, Imam Muhammad Ibn Saud Islamic University, Riyadh, Saudi Arabia \\ ${ }^{13}$ College of Medicine, Ibn Sina National College, Jeddah, Saudi Arabia
}

Received: 28 December 2020

Accepted: 13 January 2021

*Correspondence:

Dr. Abdulrahman Mohammad Ghafouri,

E-mail: dr_ghafouri@hotmail.com

Copyright: (c) the author(s), publisher and licensee Medip Academy. This is an open-access article distributed under the terms of the Creative Commons Attribution Non-Commercial License, which permits unrestricted non-commercial use, distribution, and reproduction in any medium, provided the original work is properly cited.

\begin{abstract}
This literature review aims to highlight the possible causes, complications, and management of this event from previously published studies discussing dehydration in critically-ill patients admitted to the emergency room (ER). In general, the administration of fluids has been used in the ER for critically-ill patients for many purposes. These include the optimization and adjustment of body fluids and electrolytes, increase renal protection against contrast, enhance uric acid, globins, caloric intake, and as an adjuvant to ameliorate the potentiality of certain medications or to dilute them. Many etiologies have been accused to cause dehydration in critically-ill patients. These include the fasting strategy that patients are obliged to whenever undergoing a surgical operation. Kidney, heart, and liver injuries have also been associated with patients' dehydration. Old age and the presence of other co-morbidities diabetes insipidus and uncontrolled diabetes can also aggravate the condition. Mental affection is the main complication that patients with severe dehydration might complain about. Other complications might include aggravation of heart failure, and skin diseases, and deterioration of the kidney functions and other cellular processes that require adequate nourishment for their daily normal functions. Mortality is also a serious common complication, especially within elderly patients. Although the management of dehydration can be easily achieved by fluid administration, fluid overload can aggravate the underlying complications and develop others. Therefore, the main challenge here would be to frequently monitor patients during fluid administration and resuscitation to prevent this side effect.
\end{abstract}

Keywords: Dehydration, Emergency; Complication, Management 


\section{INTRODUCTION}

Preoperative fasting before performing surgeries has been prescribed for patients undergoing surgeries to prevent any possible complications that may result from aspiration of food particles intraoperatively. ${ }^{1}$ Consequently, patients are more prone to develop a dry mouth and be hungry perioperatively. Preoperative management of these events can be easily achieved by intravenous supportive care, and by optimizing the preoperative fasting time which seems to be variable among the different healthcare systems globally. ${ }^{2}$ Based on the shortage of present scientific evidence for evaluating the theory of preoperative fasting, many approaches have been made to reevaluate this phenomenon and adjust it based on the possible outcomes. ${ }^{1}$ The relevant societies in the United States and most European countries have led such approaches to adjust the preoperative fasting guidelines and suggested that clear fluids can be orally allowed until 2-3 hours preoperatively except when a procedure for evaluating gastric emptying was planned. ${ }^{3}$

In general, strategies following restrictive fluid management approaches are encouraged during certain procedures as those involving patients with severe respiratory distress undergoing major surgical operations as they are thought to decrease the time spent on mechanical ventilation and frequency of any potential cardiopulmonary complications. ${ }^{4,5}$ Malbrain et al. ${ }^{6}$ conducted a meta-analysis to study the effect of frequency of fluid overhydration and resuscitation of critically ill patients. The authors reported that positive fluid balance was significantly associated with better outcomes regarding the patients' survival rates among the included studies. The results also demonstrated that the restrictive strategy was more significantly efficacious in reducing the mortality rates than the liberal one. ${ }^{6}$

The administration of fluids has been used in the emergency room (ER) for critically-ill patients for many purposes. These include the optimization and adjustment of body fluids and electrolytes, increase renal protection against contrast, enhance uric acid, globins, caloric intake, and as an adjuvant to ameliorate the potentiality of certain medications or to dilute them. ${ }^{7-11}$ It should be noted that the re-hydration process is a fine one and should be carefully approached. If misused, serious complications may arise as a result which is mainly attributable to overhydration that can be fatal sometimes causing cerebral edema and cardiopulmonary complications. ${ }^{12}$ In this review, we aim to discuss the different aspects of dehydration in patients admitted to the ER. We will try to highlight the possible causes, complications, and management of this event from previously published studies.

\section{METHODS}

We performed an extensive literature search of the Medline, Cochrane, and EMBASE databases on $14^{\text {th }}$ December 2020 using the medical subject headings
(MeSH) or a combination of all possible related terms. Studies reporting the causes, complications, and management of severe adult dehydration in the ER were screened for relevant information. We did not pose any limits on date, language, or publication type.

\section{DISCUSSION}

\section{Etiology of dehydration among adult patients}

It has been reported that the human kidneys lose efficacy as patients get older which is attributable to the fact that kidneys lose $33-50 \%$ of their nephrons in patients that exceed 30 years of age which affects the absorbing capacities of the kidneys. ${ }^{13,14}$ Studies also estimated that kidney function tests become heavily impaired by the age of 80 as creatinine clearance becomes $30 \%$ and serum creatinine might be elevated $300 \mathrm{~mL} / \mathrm{min}^{15}$ The same study also reported that the kidneys of older patients secrete fewer amounts of renin which affects the levels of aldosterone secretions, and consequently, the physiology of sodium and water secretion. ${ }^{15}$ In the same context, adult patients' kidneys are more prone to be less likely respondent to vasopressin which is attributable to the resistance of the osmoreceptors which increases with age. ${ }^{15-18}$ These events affect the kidneys' ability to concentrate or dilute the urine in a consequence that meets the metabolic and fluid status of the body. This increases the risk of developing hyponatremia and dehydration which is attributable to many relevant complications. As it is widely known, factors as diarrhea, diuretics, fever, heat exhaustion and cathartics are responsible for losing many metabolites and body fluids that can contribute to dehydration. In addition to these factors, organ damage, especially heart, liver, and kidney injuries and other disorders causing hormonal imbalance as inappropriate antidiuretic hormone secretion, uncontrolled diabetes, and angiotensin-converting enzyme inhibitors, contribute mainly to the process of dehydration and the disturbance in body fluids balance. Intact organ functions and appropriate hormonal secretions are responsible for body fluids and electrolyte balance within the human body. Therefore, disorders affecting this normal balance can easily cause disturbances, especially in sodium levels, which can cause a shift of fluids extracellularly and edema which can aggravate dehydration. The release of atrial natriuretic peptide (which is secreted from patients with myocardial injuries) can also aggravate dehydration by reducing the release of renin, and consequently, the activation of the renin-angiotensin cascade, causing more fluid loss. These events, in addition to other conditions as vomiting and diarrhea that seriously-ill patients, usually have, are the main causes of hypovolemia and dehydration. Drug-induced diuresis is also another factor for fluid floss and the subsequent complications in adult patients.

Sepsis is another contributing disorder to the process of dehydration. In sepsis, the body exhibits more frequent 
spells of sympathomimetic agents as adrenaline and noradrenaline which cause arteriolar vasoconstriction, including those afferent arterioles of the kidneys, which reduces the glomerular filtration rate and the kidney functions to dilute and concentrate. This will result in a state of oliguria, azotemia, and concentrate urine that is similar to that of dehydration which is also related to being infected with severe illnesses and infections. Perioperative blood loss is also a contributing factor that can be aggravated by the aforementioned etiologies. A previous study by Mukand et al reported that a significant cohort of their patients, that underwent total hip and/or knee replacement procedures, were prone to develop dehydration, azotemia, and orthostasis which was responsible for delayed healing of these patients due to the relevance of possible complications. ${ }^{19}$ Furthermore, the presence of certain comorbidities may lead to more fluid loss and complications than others. These include, in addition to renal, liver, and heart injuries, aging (as it is frequently associated with the loss of thirst sensation), dysphagia, and the underlying neurological disorders (as parkinsonism, cognitive impairment, amyotrophic lateral sclerosis) that may affect the habituation of drinking. ${ }^{20,21}$

\section{Complications and burdens of dehydration}

Many complications have been associated with the development of dehydration which affects cellular functioning and causes serious damage to many organs especially, the brain tissues. Studies showed that dehydration is accused of acute onsets of acute confusion and delirium states especially in the elderly population which lacks the synthesis of nitric oxide. ${ }^{22-25}$ The process of dehydration is responsible for increased osmolality of the plasma and the concentration of electrolytes as sodium which leads to the development of hypernatremia, and hyperosmolality. This process affects the mentality of the diseased patients as they affect the functions and release of acetylcholine which is a strong mediator for all the neurological processes. Previous studies showed that dehydration-induced complications can also aggravate more dehydration running in a vicious circle. ${ }^{26,27}$ To maintain adequate hydration is an essential need to pursue the metabolic and healing processes of an injured body. In the same context, absorption of the administered drugs also needs the availability of adequate hydration to complete the process. Chronic dehydration, therefore, would aggravate the underlying patients' conditions and delay the process of healing, and would also prolong the hospital stay. Studies showed that this can complicate many conditions and can even increase the rate of mortality, especially in the elderly. ${ }^{28,29}$ Reduced body fluids lead to reduced plasma volume which stimulates the heart to exert more efforts to increase the stroke volume to maintain adequate tissue perfusion. Disturbed thermo-regulation is another consequence as redistribution of the body fluids to the most important organs in need of adequate perfusion makes the skin vessels more liable to severe vasoconstriction causing peripheral cyanosis. ${ }^{30}$ Xiao et al. $^{31}$ also reported that dehydration might complicate other pre-existing conditions like infections, kidney stones, constipation, mitral valve prolapse, pneumonia, urinary tract infections, gastroenteritis, uncontrolled diabetes, ulcers, metabolic disturbances, and cancers. The cumulative effect of such complications will affect the patients' quality of life, especially the elderly ones which may exhibit more complications than can even cause death. A high range of $15-50 \%$ for mortality rates has been noticed among studies in the literature for institutionalized elderly patients. Mahowald and Himmelstein reported that up to $59 \%$ of their patients died as a result of dehydrationinduced pneumonia and/or urinary tract infection. Dehydration can lead to various life-threatening events as evidence shows that the rate of patients' hospitalization that is attributable to dehydration is rapidly increasing among patients within the United States.

\section{Management of dehydration and related concerns}

Although dehydration can be prevented, it is frequently reversible. ${ }^{32}$ Therefore, efforts should be directed to primarily preventing the condition by continuously monitoring patients at risk which can dramatically decrease the rate of the condition and the associated burdens. It has been reported that perioperative adequate rehydration is much more efficacious than managing post-operative dehydration. ${ }^{31}$ Although previous studies demonstrated many concerns about the screening and assessment of dehydration, recent studies have demonstrated reliable approaches for early detection and continuous monitoring of patients. ${ }^{33-35}$ History and physical examination, electrocardiogram, chest radiograph, and the serum levels of type-B natriuretic peptide (BNP) can detect the presence of severe complications that require adjusting the plan of management and resuscitation accordingly. ${ }^{36-39}$ Previous protocols were designed to decrease the preoperative fasting interval to reduce the risks of dehydration. Another approach to minimize surgery-induced stressful situations has also been approached by Fearon et al. ${ }^{40}$ namely the enhanced recovery after surgery (ERAS) aiming to reduce the demerits of perioperative fasting. Nygren et al. ${ }^{41}$ also reported that perioperative administration of fluids and carbohydrates can decrease the anxiety and the subsequent feeling of thirst and hunger that may result from perioperative fasting. Carbohydrate administration can also reduce the postoperative anticipated insulin resistance. Oral rehydration therapy (ORT) is considered one of the most efficacious modalities for the management of dehydration in such patients. As recommended by the world health organization, it can be used to compensate fluids, electrolytes, and carbohydrates which may be superior to intravenous compensation and are favored in many European countries and the United States. ${ }^{42}$ Taniguchi et al. ${ }^{43}$ conducted a cohort study to decide whether ORT is an efficacious modality for the management of dehydration in terms of efficacy and complications. The authors reported that rehydration, which means providing fluid, carbohydrates, and electrolytes could be safely 
achieved in their patients with improvements regarding the fractional excretion of $\mathrm{Na}$, kidney function tests, and volume of esophageal-pharyngeal fluid and gastric fluid. No serious complications were noticed among the study population, however, only one patient suffered from vomiting. ${ }^{43}$

Another aspect of complications that should be considered is those complications resulting from the management of patients with dehydration. For instance, fluid overload can aggravate the underlying complications and develop others. Bouchard et al. reported that the rate of recovery from a kidney injury was significantly lower in patients that suffered from fluid overload. ${ }^{44}$ In addition to the brain, the lungs are also one of the heavily affected organs by fluid imbalance. Volume overload can lead to complications as acute respiratory distress syndrome and acute pulmonary edema which can be fatal. ${ }^{45}$ Murphy et al. showed that adequate fluid therapy after resuscitation can reduce the mortality rates among hospitalized patients. ${ }^{46}$ Fluid and electrolyte balance together with a central venous pressure $>12 \mathrm{mmHg}$ are associated with better outcomes as reported by the vasopressin in septic shock trial. ${ }^{47}$ On the other hand, a previous systematic review showed that central venous pressure should not be used to decide the effectiveness of fluid management and other methods of evaluation should be approached. ${ }^{48}$

\section{CONCLUSION}

Pre-existing evidence shows that dehydration can be easily managed. However, many concerns are to be considered, especially when managing patients suffering from critical illnesses. These include the frequent monitoring of patients during fluid administration and resuscitation to prevent any possibility of adverse events causing overhydration and subsequent pulmonary and cerebral edema that can be fatal. For proper management of dehydration, special attention should be given to the etiology which should be primarily prevented to essentially eliminate the condition. Special care should also be provided for elderly patients, patients with uncontrolled diabetes, and those with underlying chronic conditions as heart, liver, or kidney diseases, and patients suffering from diabetes insipidus.

Funding: No funding sources Conflict of interest: None declared

Ethical approval: Not required

\section{REFERENCES}

1. McIntyre JW. Evolution of $20^{\text {th }}$ century attitudes to prophylaxis of pulmonary aspiration during anaesthesia. Can J Anaesth. 1998;45(10):1024-30.

2. Shime N, Ono A, Chihara E, Tanaka Y. Current practice of preoperative fasting: a nationwide survey in Japanese anesthesia-teaching hospitals. J Anesth. 2005; 19(3):187-92.

3. Søreide E, Eriksson LI, Hirlekar G, Eriksson H, Henneberg SW, Sandin R. Pre-operative fasting guidelines: an update. Acta Anaesthesiol Scand. 2005;49(8):1041-7.

4. Wiedemann HP, Wheeler AP, Bernard GR, Thompson BT, Hayden D, De Boisblanc B et al. Comparison of two fluid-management strategies in acute lung injury. N Eng j med. 2006;354(24):256475.

5. Brandstrup B, Tønnesen $\mathrm{H}$, Beier-Holgersen $\mathrm{R}$, Hjorts $\varnothing$ E, Ørding H, Lindorff-Larsen H et al. Effects of intravenous fluid restriction on postoperative complications: comparison of two perioperative fluid regimens: a randomized assessor-blinded multicenter trial. Ann Surg. 2003;238(5):641-8.

6. Malbrain M, Marik P, Witters I, Cordemans C, Kirkpatrick AW, Roberts DJ et al. Fluid overload, de-resuscitation, and outcomes in critically ill or injured patients: A systematic review with suggestions for clinical practice. Anaesthesiol intensive therapy. 2014;46:361-80.

7. Rivers E, Nguyen B, Havstad S, Ressler J, Muzzin A, Muzzin A et al. Early goal-directed therapy in the treatment of severe sepsis and septic shock. $\mathrm{N}$ Eng $\mathrm{j}$ med. 2001;345(19):1368-77.

8. Gunal AI, Celiker H, Dogukan A, Ozalp G, Kirciman E, Simsekli E et al. Early and vigorous fluid resuscitation prevents acute renal failure in the crush victims of catastrophic earthquakes. J Am Soc Nephrol. 2004;15(7):1862-7.

9. Davidson MB, Thakkar S, Hix JK, Bhandarkar ND, Wong A, Schreiber MJ. Pathophysiology, clinical consequences, and treatment of tumor lysis syndrome. Am j med. 2004;116(8):546-54.

10. Van den Berghe G, Wouters P, Weekers F, Verwaest C, Bruyninckx F, Schetz $\mathrm{M}$ et al. Intensive insulin therapy in critically ill patients. $\mathrm{N}$ Eng $\mathrm{J}$ med. 2001;345(19):1359-67.

11. Nemec K, Kopelent-Frank H, Greif R. Standardization of infusion solutions to reduce the risk of incompatibility. Am j health-system pharmacy. 2008;65(17):1648-54.

12. Besen BAMP, Gobatto ALN, Melro LMG, Maciel AT, Park M. Fluid and electrolyte overload in critically ill patients: An overview. World J Crit Care Med. 2015;4(2):116-29.

13. Kositzke JA. A question of balance--dehydration in the elderly. J gerontological nursing. 1990;16(5):411 .

14. Begum MN, Johnson CS. A review of the literature on dehydration in the institutionalized elderly. Eur eJ Clin Nutrit Metabol. 2010;5(1):e47-53.

15. Lavizzo-Mourey RJ. Dehydration in the elderly: a short review. J Natl Med Assoc. 1987;79(10):1033-8.

16. Sheehy CM, Perry PA, Cromwell SL. Dehydration: biological considerations, age-related changes, and risk factors in older adults. Biological research for nursing. 1999;1(1):30-7.

17. Phillips PA, Johnston CI, Gray L. Disturbed fluid and electrolyte homoeostasis following dehydration in elderly people. Age and ageing. 1993;22(1):S2633.

18. Martin JH, Larsen PD. Dehydration in the elderly surgical patient. Aorn j. 1994;60(4):666-71. 
19. Mukand JA, Cai C, Zielinski A, Danish M, Berman J. The effects of dehydration on rehabilitation outcomes of elderly orthopedic patients. Arch physical med rehabil. 2003;84(1):58-61.

20. Feinsod FM, Levenson SA, Rapp K, Rapp MP, Beechinor E, Liebmann L. Dehydration in frail, older residents in long-term care facilities. J Am Med Directors Asso. 2004;5(2):S35-41.

21. Mentes JC. A typology of oral hydration problems exhibited by frail nursing home residents. J gerontol nursing. 2006;32(1):13-19; quiz 20-11.

22. Mentes JC, Culp K. Reducing hydration-linked events in nursing home residents. Clinical nursing research. 2003;12(3):210-225; discussion 226-218.

23. Cacchione PZ, Culp K, Laing J, Tripp-Reimer $\mathrm{T}$. Clinical profile of acute confusion in the long-term care setting. Clin nursing res. 2003;12(2):145-58.

24. Mentes J, Culp K, Maas M, Rantz M. Acute confusion indicators: risk factors and prevalence using MDS data. Res nursing health. 1999;22(2):95105.

25. Culp K, Mentes J, Wakefield B. Hydration and acute confusion in long-term care residents. Western $\mathrm{j}$ nursing res. 2003;25(3):251-66.

26. Gaspar PM. What determines how much patients drink? Geriatric nursing (New York, NY) 1988;9(4):221-4.

27. Paulis S, Everink I, Halfens R, Lohrmann C, Schols JMGA. Prevalence and Risk Factors of Dehydration Among Nursing Home Residents: A Systematic Review. J Am Med Directors Asso. 2018;19.

28. Häussinger D, Lang F, Gerok W. Regulation of cell function by the cellular hydration state. Am $\mathrm{j}$ physiol. 1994;267(3 Pt 1):E343-55.

29. Kayser-Jones J, Schell ES, Porter C, Barbaccia JC, Shaw H. Factors contributing to dehydration in nursing homes: inadequate staffing and lack of professional supervision. J Am Geriatrics Soc. 1999;47(10):1187-94.

30. Leiter LA, Marliss EB. Survival during fasting may depend on fat as well as protein stores. Jama. 1982;248(18):2306-7.

31. Xiao H, Barber J, Campbell ES. Economic burden of dehydration among hospitalized elderly patients. Am j health-system pharmacy. 2004;61(23):2534-40.

32. Cole MG, McCusker J. Treatment of delirium in older medical inpatients: a challenge for geriatric specialists. J Am Geriatrics Soc. 2002;50(12):2101-3.

33. Thomas DR, Tariq SH, Makhdomm S, Haddad R, Moinuddin A. Physician misdiagnosis of dehydration in older adults. J Am Med Directors Asso. 2004;5(2):S30-4.

34. Kavouras SA. Assessing hydration status. Current opinion clin nutrition metabolic care. 2002;5(5):51924.

35. George J, Rockwood K. Dehydration and delirium-not a simple relationship. J Gerontol A Biol Sci Med Sci. 2004;59(8):811-2.

36. Wang CS, FitzGerald JM, Schulzer M, Mak E, Ayas NT. Does this dyspneic patient in the emergency department have congestive heart failure? Jama 2005;294(15):1944-56.

37. Butman SM, Ewy GA, Standen JR, Kern KB, Hahn E. Bedside cardiovascular examination in patients with severe chronic heart failure: importance of rest or inducible jugular venous distension. J Am College Cardiol. 1993;22(4):968-74.

38. Marantz PR, Kaplan MC, Alderman MH. Clinical diagnosis of congestive heart failure in patients with acute dyspnea. Chest. 1990;97(4):776-81.

39. Collins SP, Lindsell CJ, Storrow AB, Abraham WT. Prevalence of negative chest radiography results in the emergency department patient with decompensated heart failure. Ann emergency med. 2006;47(1):13-8.

40. Fearon KC, Ljungqvist O, Von Meyenfeldt M, Revhaug A, Dejong CHC, Lassen K. Enhanced recovery after surgery: a consensus review of clinical care for patients undergoing colonic resection. Clin nutr. 2005;24(3):466-77.

41. Nygren J, Thorell A, Jacobsson H, Larsson S, Schnell PO, Hylén L et al. Preoperative gastric emptying. Effects of anxiety and oral carbohydrate administration. Ann Surg. 1995;222(6):728-34.

42. Soop M, Nygren J, Myrenfors P, Thorell A, Ljungqvist O. Preoperative oral carbohydrate treatment attenuates immediate postoperative insulin resistance. Am j physiol Endocrinol metabol. 2001;280(4):E576-83.

43. Taniguchi H, Sasaki T, Fujita H, Takamori M, Kawasaki R, Momiyama Y et al. Preoperative fluid and electrolyte management with oral rehydration therapy. J Anesth. 2009;23(2):222-9.

44. Bouchard J, Soroko SB, Chertow GM, Himmelfarb J, Ikizler TA, Paganini EP et al. Fluid accumulation, survival and recovery of kidney function in critically ill patients with acute kidney injury. Kidney Int. 2009;76(4):422-7.

45. Schrier RW, Wang W. Acute renal failure and sepsis. N Eng j med. 2004;351(2):159-69.

46. Murphy CV, Schramm GE, Doherty JA, Reichley RM, Gajic O, Afessa B et al. The importance of fluid management in acute lung injury secondary to septic shock. Chest. 2009;136(1):102-9.

47. Boyd JH, Forbes J, Nakada TA, Walley KR, Russell JA. Fluid resuscitation in septic shock: a positive fluid balance and elevated central venous pressure are associated with increased mortality. Crit Care Med. 2011;39(2):259-65.

48. Marik PE, Baram M, Vahid B. Does central venous pressure predict fluid responsiveness? A systematic review of the literature and the tale of seven mares. Chest. 2008;134(1):172-8.

Cite this article as: Ghafouri AM, Arkoubi SH, AlAmri TM, Alshamari AM, Alqahtani MA, Alyami ZS, et al. Causes, management and complications of severe adult dehydration in the emergency room. Int J Community Med Public Health 2021;8:943-7. 